\title{
Autoimmunpankreatitis bei Vater und Sohn - 2 Fallberichte
}

\author{
Johanna Munding ${ }^{1}$, Orlin Belyaev ${ }^{2}$, Sarah R. Gerigk ${ }^{2}$, Waldemar Uhl ${ }^{2}$, Andrea Tannapfel ${ }^{1}$
}

\section{Einleitung}

Die Autoimmunpankreatitis (AIP) ist eine seltene Form der Pankreatitis und betrifft etwa $5 \%$ der Patienten mit chronischer Pankreatitis. Die Grundzüge der Entität wurden erstmals 1961 durch Sarles et al. beschrieben, die bei mehreren Patienten eine idiopathische chronische Pankreatitis mit Hypergammaglobulinämie nachweisen konnten und eine autoimmune Genese dieser Entzündung postulierten [1]. Ab 1995 - 34 Jahre später-wurde die Autoimmunpankreatitis als eigene Krankheitsentität etabliert [2].

AIP Typ I und Typ II. Aktuell erfolgt eine Differenzierung der Autoimmunpankreatitis in zwei verschiedene Typen, die von prognostischer und therapeutischer Bedeutung ist. Beide Subtypen treten bei Männern häufiger auf als bei Frauen, und Patienten mit einer Autoimmunpankreatitis Typ I sind im Durchschnitt etwa 10 Jahre älter als Patienten mit einer Autoimmunpankreatitis Typ II.

Die AIP Typ I stellt die pankreatische Manifestationsform einer generalisierten Autoimmunerkrankung dar, die mit einer Vermehrung eines spezifischen Immunglobulin-Subtyps (IgG4) einhergeht. Die AIP Typ II hingegen ist eine auf das Pankreas begrenzte Entzündung mit charakteristischem histologischem Bild, das als idiopathische duktzentrische chronische Pankreatitis mit granulozytären epithelia-

\footnotetext{
${ }^{1}$ Institut für Pathologie, Ruhr-Universität Bochum am BG-Universitätsklinikum Bergmannsheil

2 Chirurgische Klinik, St. Josef-Hospital, Ruhr-Universität Bochum
}

len Läsionen (GEL) und Eosinophilie beschrieben wird.

DD Pankreaskarzinom. Das Hauptproblem in der Diagnostik der AIP besteht in der Differenzierung zu einem Pankreaskarzinom. Besonders bei der AIP Typ I wird klinisch primär oft eine Raumforderung bzw. diffuse Vergrößerung des Pankreas gesehen, die malignitätsverdächtig imponiert und eine weitere Abklärung, häufig mit Resektion des Organs, nach sich zieht.

Im Folgenden werden zwei Patienten (Vater und Sohn) mit einer Autoimmunpankreatitis vorgestellt.

\section{Kasuistik 1 (Vater)}

\section{Anamnese und Klinik}

Der 66 Jahre alte Patient stellte sich in stabilem Allgemeinzustand und schlankem Ernährungszustand mit seit mehreren Monaten bestehender Transaminasenerhöhung und Anstieg der Cholestaseparameter vor. Bereits vorbekannt war ein Diabetes mellitus Typ 2. Ein Gewichtsverlust oder andere Beschwerden wurden nicht dokumentiert.

\section{Diagnostik und Therapie}

Bildgebung. In der weiteren bildgebenden Diagnostik zeigte sich endosonografisch ein echoarmes Pankreas mit Raumforderung im Pankreaskopf mit inhomogenem Binnenmuster. CT-morphologisch konnte jedoch keine weitere Einordnung des Befundes erfolgen. In der ERCP zeigten sich Auffälligkeiten der Gallenwege mit Stenose des intrapankreatischen Ductus choledochus und unregelmäßigen Gallenwegen intrahepatisch.

Labor. Laborchemisch bestätigte sich die Leberwerterhöhung und es zeigte sich eine Reduktion der Pankreasenzyme bei pathologischen Glukosewerten, weiterhin waren die Tumormarker CEA und CA 19-9 im Normbereich und es konnten antimitochondriale (AMA) und antiglattmuskuläre (ASMA) Antikörper nachgewiesen werden.

Punktion und Laparotomie. Zur weiteren Abklärung des Pankreaskopfprozesses erfolgte eine zweimalige endosonografisch gesteuerte Punktion des Pankreaskopfes, die lediglich entzündliche Veränderungen zeigte.

Aufgrund der klinisch als malignitätsverdächtig diskutierten Choledochusstenose wurde eine explorative Laparatomie angestrebt mit ggf. Resektion des Pankreaskopfs und einer Cholezystektomie bei koexistenter Cholezystolithiasis. Eine Choledocholithiasis lag nicht vor. Intraoperativ zeigten sich vergrößerte Lymphknoten sowie eine ausgeprägte Verhärtung des Pankreaskopfes mit Einbeziehung des intrapankreatischen Gallengangs bei sehr englumigem Pankreasgang. Es wurde eine pyloruserhaltende Whipple-Operation mit Lymphknotendissektion durchgeführt. 


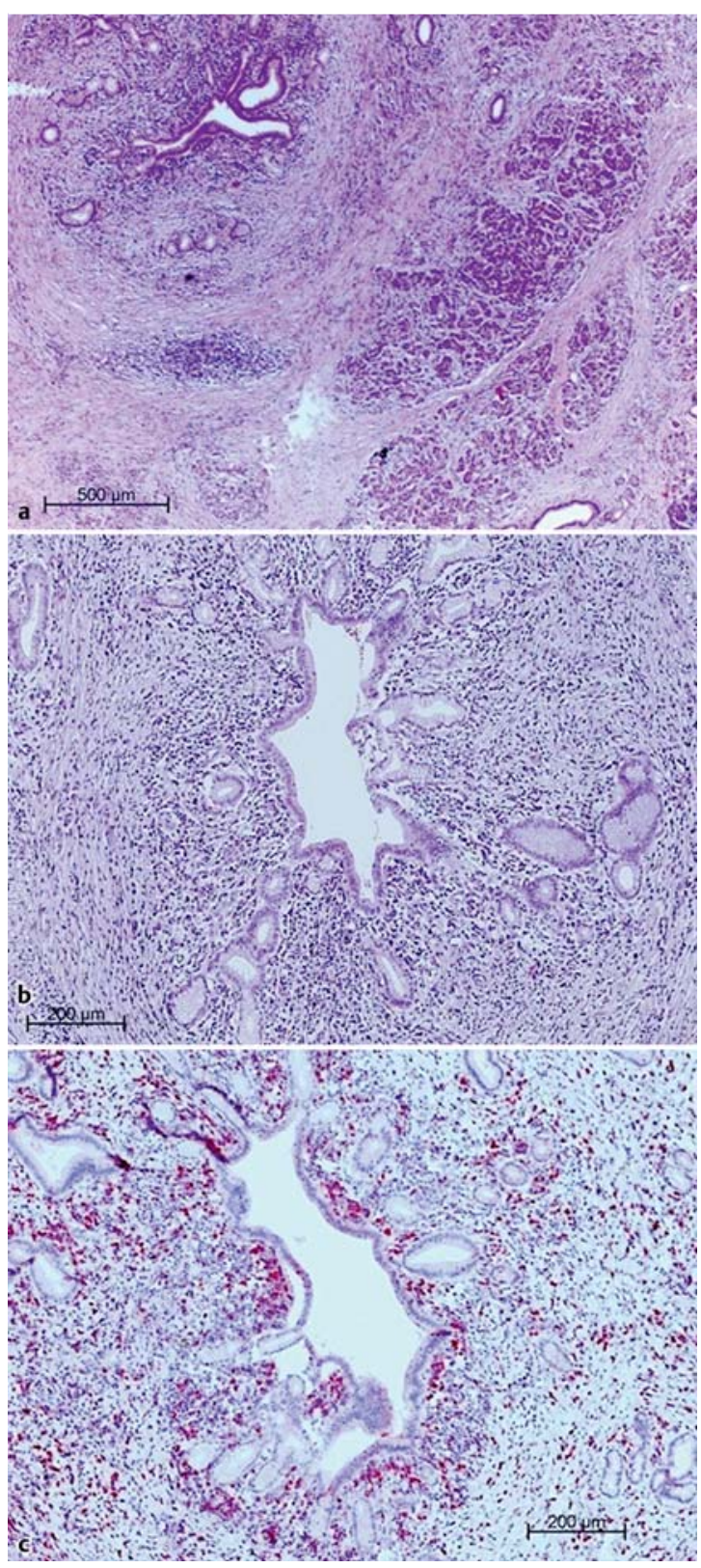

Abb. 1 Histologisches Bild bei Patient 1 (Vater). a Übersicht. b Periduktal akzentuierte Entzündung. c Vermehrung IgG4-positiver Plasmazellen immunhistochemisch.

\section{Kasuistik 2 (Sohn)}

\section{Anamnese und Klinik}

Im Juli 2013 stellte sich der damals 46 Jahre alte Sohn in gutem Allgemeinzustand und normalem Ernährungszustand vor, nachdem er mit einem seit 4 Tagen bestehenden schmerzlosen Ikterus in einem benachbarten Krankenhaus aufgenommen worden war, um eine Auffälligkeit des Pankreas in einem Pankreaszentrum weiter abklären zu lassen. An Vorerkrankungen waren ein Asthma bronchiale und mehrfache Operationen an den Nasennebenhöhlen bekannt.

\section{Diagnostik und Therapie}

Zytologie. Im Aufnahmekrankenhaus war bereits eine Endosonografie mit Punktion des Pankreas erfolgt und der Verdacht auf ein Pankreaskarzinom gestellt worden. Punktionszytologisch ergab sich keine wegweisende Diagnose. Es konnten zytologisch atypische Zellkomplexe vor einem entzündlichen Hintergrund nachgewiesen werden. Zusammenfassend wurde der zytologische Befund als schwere chronische Pankreatitis gewertet, mit nicht eindeutig nachweisbaren Karzinomzellen, aber dringendem weiterem Klärungsbedarf.

CT. CT-morphologisch (Abb. 2) zeigte sich eine dringend malignitätsverdächtige Pankreasraumforderung mit Kompression des Ductus choledochus mit konsekutivem Gangaufstau, differenzialdiagnostisch schien eine chronische Pankreatitis aufgrund fehlender Begleitreaktion des peripankreatischen Gewebes eher weniger wahrscheinlich. problemlos und der Patient konnte zeitgerecht entlassen werden. Die empfohlenen Nachsorgeuntersuchungen konnten bei Incompliance des Patienten leider nicht durchgeführt werden. Aktenkundig sind zuletzt 2011 und 2013 weitere Krankenhausaufenthalte des mittlerweile 76jährigen Patienten im Rahmen einer Pneumonie und zur Implantation eines Schrittmachers bei Vorhofflimmern.
ERCP und MRCP. In der durchgeführten ERCP zeigte sich eine filiforme Stenose des distalen Gallengangs über $20 \mathrm{~mm}$, etwa $20 \mathrm{~mm}$ proximal der Ampulle, die malignitätsverdächtig imponierte und zunächst durch Bougierung und Stenteinlage zur Ikterustherapie behandelt wurde. Im Verlauf waren die Cholestaseparameter 


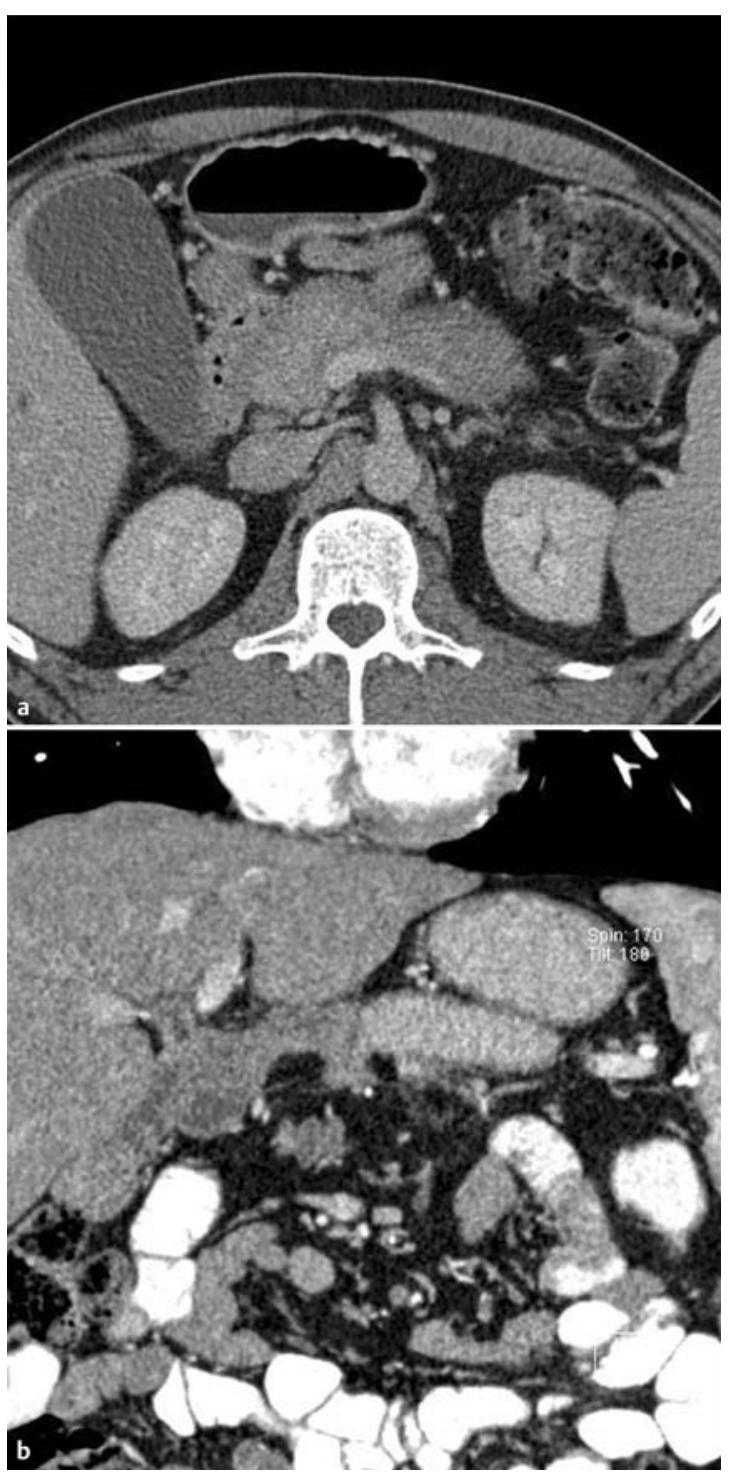

rückläufig. In der MRCP (Abb. 3) war ein Aufstau des Ductus hepaticus communis gesehen worden, während der Pankreasgang fast nicht darstellbar war. Ein sog. „Double Duct Sign“ lag nicht vor. Die Tumormarker CEA und CA 19-9 waren gering erhöht. Autoantikörper waren nicht nachweisbar.

Laparotomie. Bei dem Patienten wurde zur weiteren Abklärung und Therapie eine explorative Laparotomie mit ggf. Pankreaskopfresektion geplant.

Intraoperativ zeigten sich peripankreatisch entzündlich verändertes Gewebe sowie vergrößerte Lymphknoten und ein aufgetriebenes Pankreas mit deutlichen
Abb. 2 CT-Befunde bei Patient 2 (Sohn). a Präoperativ mit diffuser Organvergrößerung in der venösen Phase. $\mathbf{b}$ Ein Jahr postoperativ mit Darstellung der Pankreatikojejunostomie mit nach wie vor vergrößertem Pankreasschwanz.

\section{Histologie}

Die weitere pathohistologische Aufarbeitung (Abb.4a) zeigte eine schwere chronische Pankreatitis des Pankreaskopfs mit Ausbildung eines Pankreaspseudotumors, wobei sich eine Beteiligung des intrapankreatischen Ductus choledochus und des peripankreatischen Fettgewebes (Abb.4c) nachweisen ließ. Es fand sich ein typisches histologisches Bild mit storiformer Fibrose, Reduktion der azinären Strukturen und einem dichten lymphoplasmazellulären Entzündungsinfiltrat mit einzelnen Lymphfollikeln periduktal. Der Ductus pancreaticus und die kleinen Ductuli zeigten enge Lumina mit monomorpher epithelialer Auskleidung mit akzentuierter periduktaler lymphoplasmazelluärer Entzündungsreaktion ohne Nachweis von Konkrementen.

Immunhistochemie. Die immunhistochemische Untersuchung ergab eine Vermehrung IgG4-positiver Plasmazellen (Abb.4b), sodass die Diagnose einer Autoimmunpankreatitis Typ I gestellt wurde. Bei präoperativ suspekter Zytologie und klinisch auffälligem Befund wurde zudem auch der Ductus choledochus ausgedehnt aufgearbeitet. Es konnte eine Beteiligung im Rahmen der IgG4-assoziierten Erkrankung nachgewiesen werden mit additiven Veränderungen, die zur vorbeschriebenen Galleabflussstörung mit Stenteinlage passen (Regeneratepithel).

chronisch-entzündlichen Veränderungen, die das umgebende Gewebe mit einbezogen und eine Präparation erschwerten. Ein solitärer Tumor war intraoperativ nicht tastbar, der Pankreasgang sehr englumig und schwer sondierbar. Der Ductus choledochus imponierte dilatiert und deutlich wandverdickt. Im Rahmen der intraoperativen Schnellschnittdiagnostik zeigten sich tumorfreie Resektionsränder und es konnten zunächst hochgradige entzündliche Pankreasveränderungen nachgewiesen werden.

\section{Verlauf}

Der postoperative Verlauf gestaltete sich bei Magen-Darm-Atonie etwas prolongiert, in den weiteren Nachkontrollen konnte eine persistierende IgG4-Vermehrung serologisch nachgewiesen werden, und der Patient wurde zunächst mit $40 \mathrm{mg}$ Hydrocortison und anschließend abnehmender, ausschleichender Dosierung behandelt.

Im Rahmen der Nachsorge zeigten sich aktuell Gangveränderungen des Ductus pancreaticus im Pankreasschwanz, die zur 

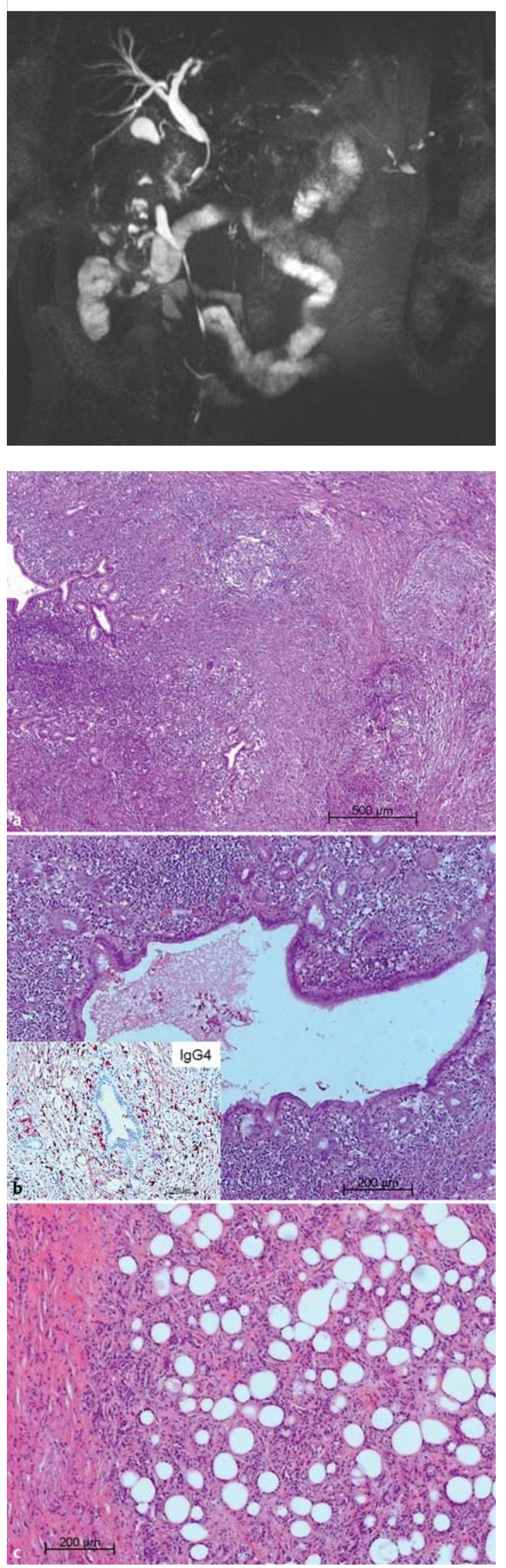

Abb. 3 MRCP nach DHC-Stentanlage bei Patient 2 (Sohn) mit Dilatation der Gallenwege. Patient 2 (Sohn). a Übersicht. b Periduktale Entzündung und Inset mit immunhistochemischem IgG4Nachweis im periduktalen Entzündungsinfiltrat. c Beteiligung des umgebenden Fettgewebes. vorbekannten AIP Typ I passen. Allerdings hat der Patient keine Beschwerden und zeigt keine Hinweise auf eine generalisierte IgG4-assoziierte Erkrankung. Zur Therapie einer exokrinen Pankreasinsuffizienz nimmt er Kreon ein, weist jedoch keine endokrine Pankreasinsuffizienz auf (unauffälliger oraler Glukosetoleranztest).

\section{Diskussion}

\section{Klinik und Befunde}

Beide Fallberichte zeigen typische Befunde, wie sie zunächst präoperativ sowohl bei einem malignen Pankreastumor als auch bei einer chronischen Pankreatitis, insbesondere einer Autoimmunpankreatitis, auftreten. Beide Patienten stellten sich mit einer Ikterussymptomatik vor, zeigten eine suspekte Pankreasraumforderung ohne eindeutige Malignitätskriterien und hatten zunächst keine weiteren Risikofaktoren für ein Pankreaskarzinom. Sie waren Nichtraucher ohne vorbestehende Pankreatitis und es lagen auch keine anderen relevanten Vorerkrankungen oder eine Alkoholanamnese vor.

Dies sind zunächst sehr weiche Kriterien, die gegen die Diagnose eines malignen Tumors sprechen. Aktuelle Zahlen belegen, dass die Inzidenz einer benignen Pankreaserkrankung bei aus Karzinomverdacht durchgeführter Pankreasresektion etwa $13 \%$ beträgt und 30-43\% dieser Patienten, die zunächst-wie die beiden oben genannten Patienten - unter Karzinomverdacht operiert wurden, an einer Autoimmunpankreatitis erkrankt sind [3].

\section{Kriterien für eine AIP. Weitere Kriterien,} die bei einer präoperativen Diagnosestellung einer AIP helfen sollen, wurden in den letzten Jahren erarbeitet $[4,5]$. So imponiert eine AIP in der Bildgebung als diffuse Organvergrößerung („wurstartig“, Abb.5) mit nur englumigem Pankreasgang ohne prästenotische Dilatation oder kalkdichte Konkremente. Laborchemisch können eine Hyperimmunglobulinämie und besonders eine IgG4-Vermehrung vorlie- 


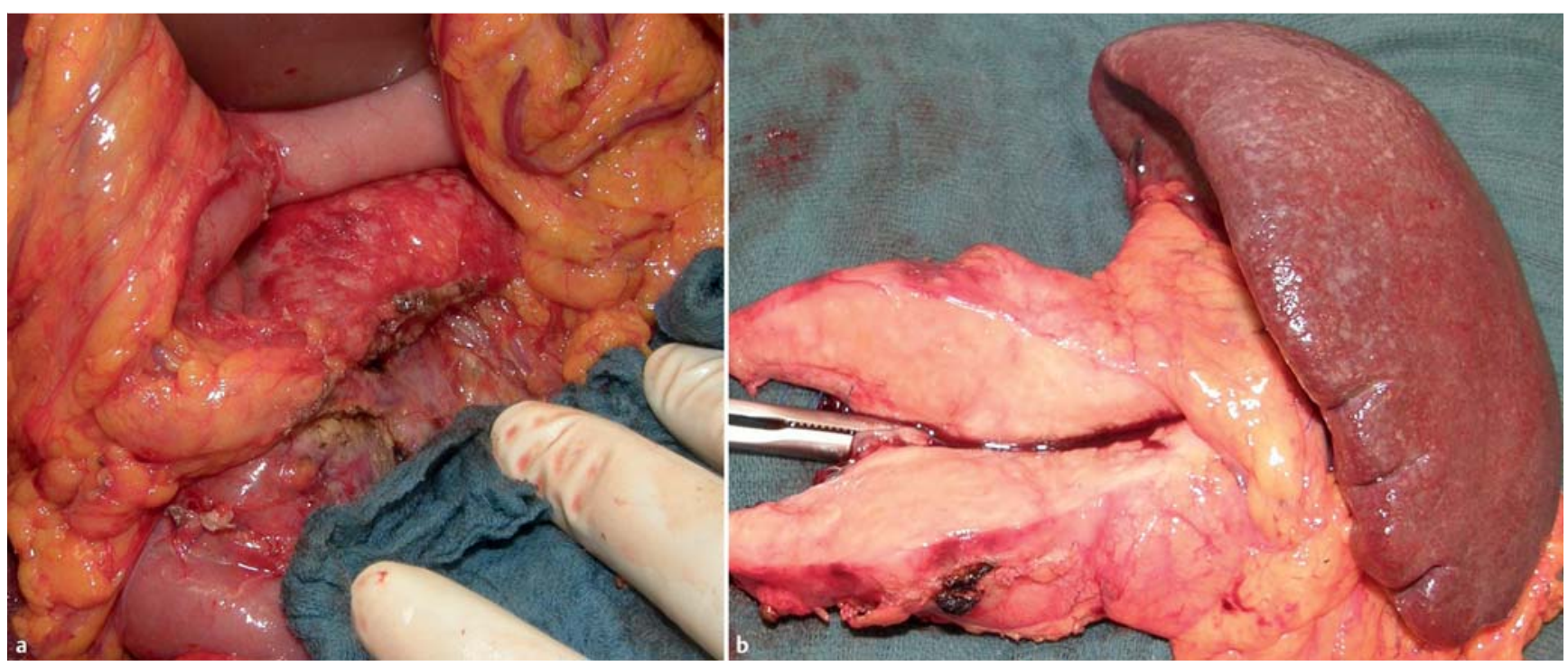

Abb.5 Operationspräparat eines Patienten (anderer Fall) mit einer AIP Typ I mit typischer „wurstartiger“ Vergrößerung des Pankreas, hier betont des Pankreasschwanzes, sodass die Indikation zur Linksresektion bestand. a Operationssitus (bei geplanter Linksresektion). b Befund nach Resektion des Pankreasschwanzes mit Milz vor Übersendung in die Pathologie zur Schnellschnittuntersuchung.

gen, und klinisch besteht eine kurzdauernde Symptomatik mit Ikterus, Oberbauchbeschwerden und möglicherweise neu aufgetretenem Diabetes mellitus. Besonders bei der AIP Typ I ist eine Beteiligung anderer Organe möglich. Eine Sicca-Symptomatik mit Sialadenitis, ein Sjögren-Syndrom oder auch die retroperitoneale Fibrose ebenso wie eine IgG4-assoziierte sklerosierende Cholangitis können im Rahmen einer IgG4-assoziierten Erkrankung vorkommen. Demgegenüber kann eine AIP Typ II auch assoziiert mit einer chronisch entzündlichen Darmerkrankung (CED) auftreten. So haben 16 $30 \%$ der Patienten mit AIP Typ II eine CED, häufiger eine Colitis ulcerosa.

Biopsie und Steroidtherapie. Steht die Verdachtsdiagnose einer Autoimmunpankreatitis zur Diskussion, so sollte eine bioptische Diagnosesicherung angestrebt werden, wobei dem Pathologen unbedingt der Verdacht auf eine Autoimmunpankreatitis mitgeteilt werden muss. Nur so kann eine schnelle immunhistochemische differenzialdiagnostische Abklärung erfolgen, und auch zytologisch ist der Nachweis vermehrter IgG4-positiver Plasmazellen möglich. Das letzte Merkmal des unter HISORt als Akronym (Histology, Imaging, Serology, Other organ involve- ment and Response to therapy) zusammengefassten Algorithmus betrifft eine probatorische Steroidtherapie über 2 Wochen [6]. Insgesamt besteht bei beiden Typen der AIP eine hohe Steroidsensitivität mit einem Ansprechen in über $98 \%$ bei der AIP Typ I und etwas geringeren

Responseraten bei der AIP Typ II. Der Therapieversuch erfolgt mit 0,6-1 mg Prednisolon pro kg Körpergewicht bei starken Indizien für eine Autoimmunpankreatitis [7]. Eine Studie konnte hier keinen Nachteil für Patienten nachweisen, bei denen dann nach Resektion doch ein Karzinom diagnostiziert wurde [8].

\section{Fazit}

Die beiden Fälle sollen die klinische Problematik bei der Abklärung einer unklaren Pankreasraumforderung illustrieren.

Zusammenfassend ist ein benigner Befund bei zunächst unter Malignitätsverdacht operiertem Patienten nicht selten und kommt in bis zu $13 \%$ der Fälle vor. Besonders wichtig für eine gute präoperative Diagnostik ist die interdisziplinäre Zusammenarbeit. Primär werden die Patienten bei Ikterus internistisch zur Abklärung aufgenommen und dann an den Chirurgen zur Diagnosesicherung und chirurgisch-onkologischen Therapie

\section{Pathogenese und Genetik}

Vater und Sohn zeigten ähnliche Krankheitsverläufe. Der Verdacht auf eine familiäre Prädisposition/Vererbung liegt nahe, wurde aber auch aufgrund mangelnder Compliance der Patienten im vorliegenden Fall nicht weiter verfolgt.

Hereditäre Assoziation. Bekannt ist, dass wie bei anderen Autoimmunerkrankungen auch bei der AIP eine hereditäre Assoziation vorliegen kann. Es scheint eine Prädisposition bei bestimmten Variationen des MHC-II-Gens vorzuliegen. Für

überwiesen. Eine genaue Patientenübergabe mit sorgfältiger Analyse der Laborwerte und der bildgebenden Diagnostik sollte im Vordergrund stehen und im Verdachtsfall eine kurze probatorische Steroidtherapie über 2 Wochen erwogen werden. Hier liefern die oben beschriebenen HISORt-Kriterien einen Anhaltspunkt. Die histologische Diagnosesicherung sollte auch an bioptischem, endosonografisch gewonnenem Material versucht werden. Dem Pathologen sind ebenfalls genaue Informationen zur Klinik zu übermitteln. 
eine Assoziation mit bestimmten HLAHaplotypen, wie DRB1*0405/DQB1*0401 oder ABCF1, der MHC-I-Gene liegen bisher Daten vor, die partiell im Tiermodell verifiziert wurden $[9,10]$.

Autoantikörper. In den Seren der betroffenen Patienten konnten zudem gehäuft Autoantikörper gegen Lactoferrin, Carboanhydrase, Ubiquitin-Ligase, Trypsin oder PSTI (Pancreatic secretory Trypsin Inhibitor) nachgewiesen werden sowie auch gegen bestimmte Helicobacter-pyloriAntigene. Pathogenetisch wurde so auch ein molekulares Mimikry diskutiert. Allerdings ist nicht abschließend geklärt, welche Autoantikörper pathogenetisch relevant sein könnten oder ob lediglich Epiphänomene vorliegen [11,12].

Interessenkonflikt: Die Autoren erklären, dass kein Interessenkonflikt besteht.

\section{Literatur}

1 Sarles H, Sarles JC, Muratore R et al. Chronic inflammatory sclerosis of the pancreas - an autonomous pancreatic disease? Am J Dig Dis 1961; 6: $688-698$

2 Yoshida K, Toki F, Takeuchi T et al. Chronic pancreatitis caused by an autoimmune abnormality. Proposal of the concept of autoimmune pancreatitis. Dig Dis Sci 1995; 40: 1561 1568

3 Asbun HJ, Conlon K, Fernandez-Cruz L et al. When to perform a pancreatoduodenectomy in the absence of positive histology?. A con- sensus statement by the International Study Group of Pancreatic Surgery Surgery 2014; 155: $887-892$

4 Okazaki K, Tomiyama T, Mitsuyama T et al. Diagnosis and classification of autoimmune pancreatitis. Autoimmun Rev 2014; 13: 451 458

5 Deshpande V, Zen Y, Chan JK et al. Consensus statement on the pathology of IgG4-related disease. Mod Pathol 2012; 25: 1181 - 1192

6 Agrawal S, Daruwala C, Khurana J. Distinguishing autoimmune pancreatitis from pancreaticobiliary cancers: current strategy. Ann Surg 2012; 255: $248-258$

7 Beyer G, Menzel ], Kruger PC et al. [Autoimmune pancreatitis]. Dtsch Med Wochenschr 2013; 138: 2359-2370 ; Quiz 2371-2374

8 Moon SH, Kim MH, Park DH et al. Is a 2-week steroid trial after initial negative investigation for malignancy useful in differentiating autoimmune pancreatitis from pancreatic cancer? A prospective outcome study Gut 2008; 57: $1704-1712$

9 Ota M, Katsuyama Y, Hamano $\mathrm{H}$ et al. Two critical genes (HLA-DRB1 and ABCF1)in the HLA region are associated with the susceptibility to autoimmune pancreatitis. Immunogenetics 2007; 59: 45-52

10 Freitag TL, Cham C, Sung $\mathrm{HH}$ et al. Human risk allele HLA-DRB $1{ }^{*} 0405$ predisposes class II transgenic Ab0 NOD mice to autoimmune pancreatitis. Gastroenterology 2010; 139: $281-291$

11 Kamisawa T, Chari ST, Lerch MM et al. Recent advances in autoimmune pancreatitis: type 1 and type 2. Gut 2013; 62: 1373-1380

12 Lohr JM, Faissner R, Koczan D et al. Autoantibodies against the exocrine pancreas in autoimmune pancreatitis: gene and protein expression profiling and immunoassays identify pancreatic enzymes as a major target of the inflammatory process. Am J Gastroenterol 2010; 105: 2060 - 2071

\section{Korrespondenzadresse}

Dr. med. Johanna Munding Institut für Pathologie

Ruhr-Universität Bochum am BGUniversitätsklinikum Bergmannsheil Bürkle-de-la-Camp-Platz 1 44789 Bochum E-Mail: johanna.munding@ruhr-unibochum.de 